\title{
Cryoplanation littorale et cordons glaciels de basse mer dans la région de Rimouski, côte sud de l'estuaire du Saint-Laurent, Québec
}

\author{
Coastal Cryoplanation and boulder barricades in the Rimouski \\ area, south shore of the St. Lawrence Estuary, Québec \\ Küsten Kryoplanation und Felsblock Barrikaden in der \\ Rimouski Gegend, Süd-Küste der Mündung des Skt Lorenz \\ Stromes, Québec
}

André Guilcher

Volume 35, numéro 2, 1981

Morphologie littorale et marine

URI : https://id.erudit.org/iderudit/1000434ar

DOI : https://doi.org/10.7202/1000434ar

Aller au sommaire du numéro

Éditeur(s)

Les Presses de l’Université de Montréal

ISSN

0705-7199 (imprimé)

1492-143X (numérique)

Découvrir la revue

Citer cet article

Guilcher, A. (1981). Cryoplanation littorale et cordons glaciels de basse mer dans la région de Rimouski, côte sud de l'estuaire du Saint-Laurent, Québec. Géographie physique et Quaternaire, 35(2), 155-169.

https://doi.org/10.7202/1000434ar
Résumé de l'article

Des plates-formes de cryoplanation développées dans des schistes ordoviciens dans la région de Rimouski sont décrites. La cryoplanation, étroitement liée à l'action de la glace de mer pendant le long hiver, est active dans les parties supérieure et moyenne de l'estran rocheux, notamment à la pointe à la Cive, au promontoire de l'Orignal, au cap du Caribou, à la pointe aux Cenelles, et, à un moindre degré, à la pointe Mitis. La nature et le pendage vertical des schistes constituent des facteurs favorables. Une cryoplanation littorale s'est également exercée au cours de l'Holocène au droit de la terrasse Mic Mac, alias Mitis, sise en arrière du rivage actuel. Elle a succédé à des agents de planation antérieurs qui ont tronqué des parties de crêtes appalachiennes sur les rives de l'estuaire du Saint-Laurent, et est remplacée par des processus marins azonaux au niveau de basse mer. Là où elle a été observée, c'est certainement un puissant facteur d'érosion. Des cordons de gros blocs rencontrés au niveau des basses mers sont décrits dans la seconde partie. Le site principal, à

Sacré-Coeur-de-Rimouski, est décrit en détail et sert de point de référence pour les autres sites de la côte sud. Des observations faites à l'époque de la débâcle montrent que ces cordons de basse mer sont dus à des apports de cailloux par radeaux de glace échoués à ce niveau parce qu'ils ne peuvent atteindre le haut de l'estran alors occupé par un pied de glace résiduel. Cette explication diffère de celle proposée pour expliquer les cordons glaciels de basse mer le long de la côte du Labrador.
Tous droits réservés @ Les Presses de l’Université de Montréal, 1981
Ce document est protégé par la loi sur le droit d'auteur. L’utilisation des services d'Érudit (y compris la reproduction) est assujettie à sa politique d'utilisation que vous pouvez consulter en ligne.

https://apropos.erudit.org/fr/usagers/politique-dutilisation/ 


\section{CRYOPLANATION LITTORALE ET CORDONS GLACIELS DE BASSE MER DANS LA RÉGION DE RIMOUSKI, COTTE SUD DE L'ESTUAIRE DU SAINT-LAURENT, QUÉBEC}

André GUILCHER, Institut de géographie, université de Bretagne occidentale, b.p. 860, 29279 Brest cedex, France.

ABSTRACT Coastal Cryoplanation and boulder barricades in the Rimouski area, south shore of the St. Lawrence Estuary, Québec. Present-day cryoplanation platforms occurring in Ordovician shales on either side of Rimouski are described in the first part of the paper. Cryoplanation closely related to sea-ice action during the longlasting winter is in progress on the higher and middle parts of intertidal platforms, particularly at Pointe à la Cive. Orignal Promontory, Cap du Caribou, Pointe aux Cenelles, and, to a smaller extent, Pointe Mitis. The nature and vertical dip of the shales are favourable factors. Coastal cryoplanation has also been efficient on the Holocene, now uplifted, Mic Mac alias Mitis terrace located behind the present shoreline. It followed previous planation agents which truncated parts of the Appalachian ridges on the banks of the St. Lawrence Estuary, and it is replaced by azonal marine processes at low-tide level. In the places where it has been observed, it is certainly a powerful factor of erosion. Boulder barricades, or boulder ridges, occurring at low-tide level are discussed in the second part of the paper. The site of Sacre-Cceur-de-Pimouski is described in detail and serves as a reference site, while several other sites are also reported. According to observations made during the breakup season, these boulder barricades result from the melting of icerafts carrying rocky blocks that are unable to reach the upper part of the intertidal zone still covered by a residual icefoot. This explanation differs from that suggested for boulder barricades occurring along the Labrador coastline.
RÉSUME Des plates-formes de cryoplanation développées dans des schistes ordoviciens dans la région de $\mathrm{Ri}$ mouski sont décrites. La cryoplanation, étroitement liée à l'action de la glace de mer pendant le long hiver, est active dans les parties supérieure et moyenne de l'estran rocheux, notamment a la pointe a la Cive, au promontoire de I'Orignal, au cap du Caribou, a la pointe aux Cenelles, et, à un moindre degré, a la pointe Mitis. La nature et le pendage vertical des schistes constituent des facteurs favorables. Une cryoplanation littorale s'est également exercée au cours de l'Holocene au droit de la terrasse Mic Mac, alias Mitis, sise en arrière du rivage actuel. Elle a succédé à des agents de planation antérieurs qui ont tronqué des parties de crétes appalachiennes sur les rives de l'estuaire du Saint-Laurent, et est remplacée par des processus marins azonaux au niveau de basse mer. Là où elle a été observée, c'est certainement un puissant facteur d'érosion. Des cordons de gros blocs rencontrés au niveau des basses mers sont decrits dans la seconde partie. Le site principal, à Sacré-Coeur-deRimouski, est décrit en détail et sert de point de réference pour les autres sites de la côte sud. Des observations faites à l'époque de la débâcle montrent que ces cordons de basse mer sont dus a des apports de cailloux par radeaux de glace échoués a ce niveau parce qu'ils ne peuvent atteindre le haut de l'estran alors occupé par un pied de glace résiduel. Cette explication differe de celle proposée pour expliquer les cordons glaciels de basse mer le long de la côte du Labrador.
ZUSAMMENFASSUNG Küten Kryoplanation und Felsblock Barrikaden in der Rimouski Gegend, Süd-Küste der Mündung des Skt Lorenz Stromes, Québec. Kryoplanation, in engem Zusammenhang mit der See-Eis-Wirkung wăhrend des lang anhaltenden Winters stehend, ist im Vorschreiten auf den höheren und mittleren Teilen der Gezeitensaum-Plattform, besonders bei Pointe à la Cive, Promontoire Orignal, Cap du Caribou, Pointe aux Cenelles, und in minderem Aussmass an der Pointe de Mitis. Die Natur und der senkrechte Neigungswinkel der Schiefer sind begünstigende Einflüsse. KüstenKryoplanation hat auch im Holozăn an der Mic Mac, allas Mitis Terrasse, welche sich hinter dem heutigen Ufer befindet, stattgefunden. Sie folgte fròheren Planationsprozesse, welche die Appalachen Gipfel an den Mündungsufern des Skt Lorenz Stromes abgestumpft haben und wurde durch azonale Meeresvorgănge an der Ebbelinie erstezt. An den beobachteten Orten, ist sie bestimmt eine mâchtige Erosionskraft. Felsblock Barrikaden und-walle die an der Ebbelinie vorkommen, werden im zweiten Teil der Arbeit behandelt. Der Hauptort, Sacré Coour de Rimouski ist genau beschrieben und dient als Beispiel für alle anderen Orte der Sûdküste. Beobachtungen zur Zeit des Eisbruches, zeigen, dass die felsblock Barrikaden durch das Tauen von Eisflossen entstehen, die Felsblöcke tragen und welche den oberen Teil des Gezeitensaumes auf Grund der Decke des residuellen Eisfusses nicht erreichen kőnnen. Diese Beobachtung unterscheidet sich von der fur Felsblock Barrikaden an der Ebbelinie gefundenan Erklărung an der Labrador Küste. 


\section{INTRODUCTION}

Depuis les travaux de BROCHU (1961) et surtout de DIONNE (1968, 1969, 1970, 1972a et c, 1979), on sait que les rivages de l'estuaire du Saint-Laurent sont le siège de processus glaciels qui, spécialement sur la côte sud, apportent sur les schorres par radeaux de glace des blocs rocheux pouvant atteindre plusieurs mètres cubes, et $y$ arrachent des paquets de végétation herbacée avec leurs enracinements vaseux pour les déposer ailleurs, en laissant des mares comme traces de cette ablation. Un engel littoral qui sur la côte sud débute en général en novembre, et qui ne s'achève qu'en avril avec parfois des débâcles momentanées; une glace côtière qui atteint finalement une épaisseur minimale de $50 \mathrm{~cm}$, moyenne de $75 \mathrm{~cm}$, et peut dépasser $1 \mathrm{~m}$; des vents forts susceptibles d'entrainer des dérives de glaces à travers tout l'estuaire et d'accumuler dans la région appalachienne du sud de très nombreux éléments du socle précambrien du nord: tout cela a été bien dit, et nous nous contentons de le rappeler brièvement, en ajoutant que, dès la fin d'octobre (par exemple, le 28 octobre en 1980), la mer peut geler dans les mares hautes de l'estran de la région de Rimouski, et dès le début de novembre dans les mares de moyen estran, ce qui fait six bons mois d'actions cryergiques littorales. Par ailleurs, il y a possibilité de chutes de neige pendant huit mois (VILLENEUVE, 1967).

L'objectif de cette contribution est de décrire, de part et d'autre de Rimouski entre Grand-Métis et SaintFabien, sur une longueur d'environ $75 \mathrm{~km}$ (fig. 1), deux types morphologiques littoraux liés au gel hivernal du rivage et à ses modalités, observés en 1973, 1975 et 1980: d'une part, une cryoplanation littorale favorisée par la lithologie et le mode d'affleurement de l'Ordovicien; d'autre part, l'édification de cordons de blocs glaciels à l'étage de la basse mer.

En cette région, la courbe de marée de Pointe-auPère montre que l'on est en présence du type mixte à prédominance semi-diurne (rapport $\frac{\mathrm{K} 1+\mathrm{O} 1}{\mathrm{M} 2+\mathrm{S} 2}$ compris entre 0,25 et 1,5 ), dans lequel il y a de petites et de grandes hautes mers à l'intérieur des grandes marées et

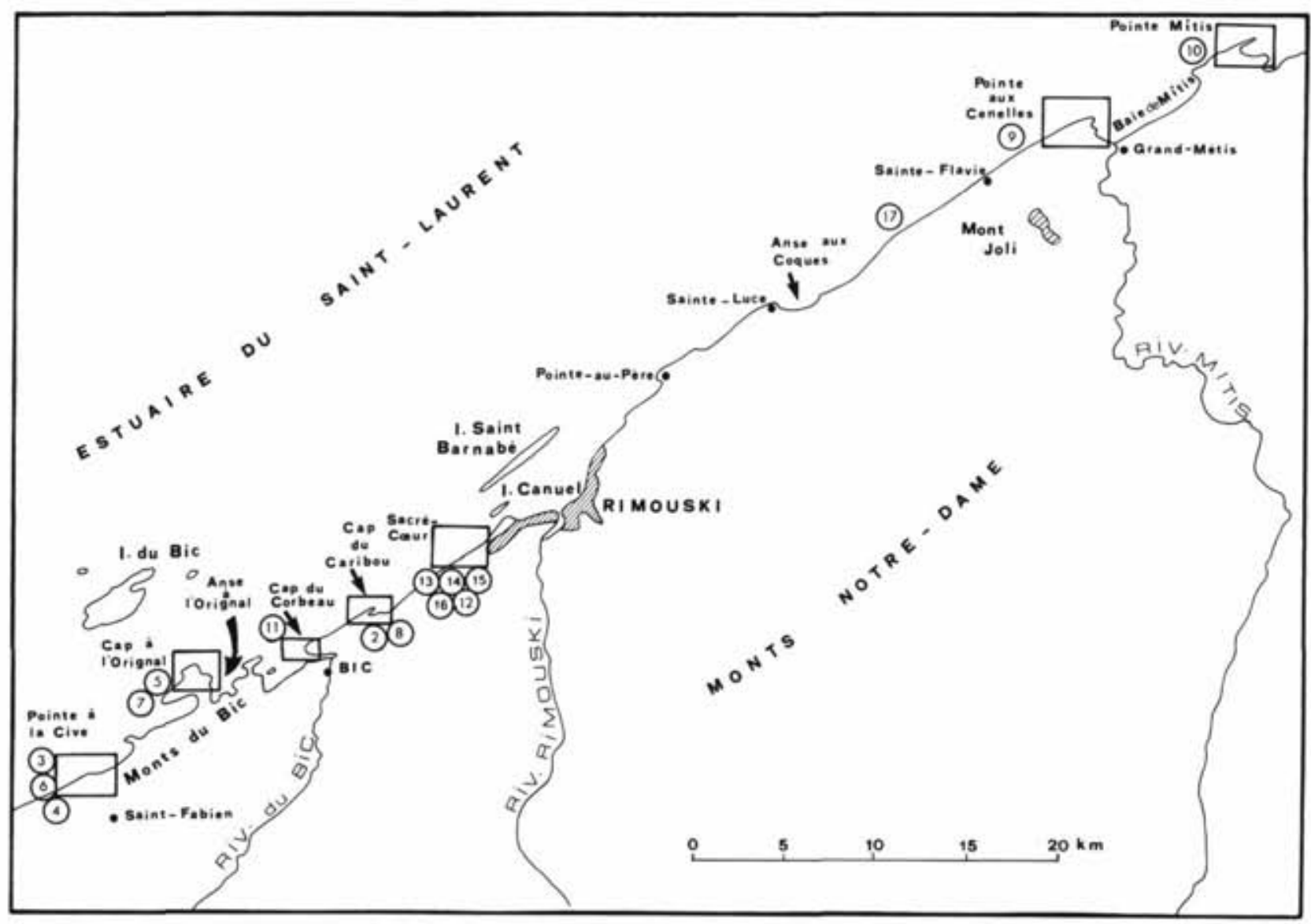

FIGURE 1. Localisation générale des lieux d'étude dans la région de Rimouski. Encadrements: emplacements des figures au trait et des phoGeneral location map, indicating places and picture numbers. tos, avec leurs numeros. 
des mortes eaux. L'inégalité des hautes mers successives peut atteindre et même dépasser $0,60 \mathrm{~m}$, tant en morte eau qu'en grande marée; en très grande marée, le marnage peut aller jusqu'à $4,70 \mathrm{~m}$, et en morte eau il peut s'abaisser en-dessous de $1,70 \mathrm{~m}$, entre la grande haute mer et la grande basse mer dans les deux cas.

\section{CRYOPLANATION LITTORALE}

\section{LE CADRE MORPHOSTRUCTURAL}

La rive sud de l'estuaire maritime du Saint-Laurent (un des plus vastes du monde, bras de mer plutôt qu'estuaire, mais avec des fetches pourtant limités du type des mers bordières et non des océans) est constituée géologiquement par un élément du système des Appalaches, orienté SO-NE, qui comprend des terrains ordoviciens et peut-être partiellement cambriens (POOLE et al., 1970 ; LAJOIE et al., 1974), très plissottés et affleurant le plus souvent de façon subverticale. Les faciès les plus fréquents sont des schistes en lames fines, des grès et grauwackes en bancs plus épais, de l'ordre de quelques centimètres, des argilites, et localement des calcaires conglomératiques. L'essentiel à retenir est la grande fréquence des faciès schisteux finement lités et des affleurements subverticaux. La différenciation lithologique et les plissements orientés SO-NE ont conduit à la réalisation de reliefs appalachiens, de présence normale en cette région éponyme, qui vont des micro-topographies en arêtes de l'ordre du mètre ou même moins, fort caractéristiques sur tous les estrans, à des crêtes et sillons classiques de l'ordre de la centaine de mètres, les crêtes culminant, pour l'aire limitée qui nous concerne, à $345 \mathrm{~m}$ au mont du Bic, à $1100 \mathrm{~m}$ seulement de distance de la mer; mais l'énergie du relief est généralement moins grande qu'à cet endroit. Les alignements sont constants et très serrés, et jouent un rôle fondamental dans le relief continental et littoral (DIONNE, 1963a).

L'héritage pléistocène est celui de la glaciation, moins importante en elle-même qu'en sa séquelle de la mer holocène de Goldthwait (DIONNE, 1977) qui, lors de sa récession, a façonné la " terrasse Mic Mac " (GOLDTHWAIT, 1911; JOHNSON, 1925, p. 224-234), ou * terrasse Mitis " (DIONNE, 1963b), courant tout au long du rivage à peu de mètres au-dessus du niveau marin actuel, et limitée à l'intérieur par une falaise morte très fraiche. II y aura lieu de se demander en quelle mesure une partie externe de cette terrasse n'a pas préfiguré la plateforme rocheuse littorale actuelle.

\section{LES FAITS ESSENTIELS}

Dans la région concernée, la gélifraction intertidale a déjà été remarquée aux environs du Bic (CAILLEUX et HAMELIN, 1967, p. 363). Elle affecte essentiellement les schistes finement lités et subverticaux (fig. 2 et 3 ), attei-

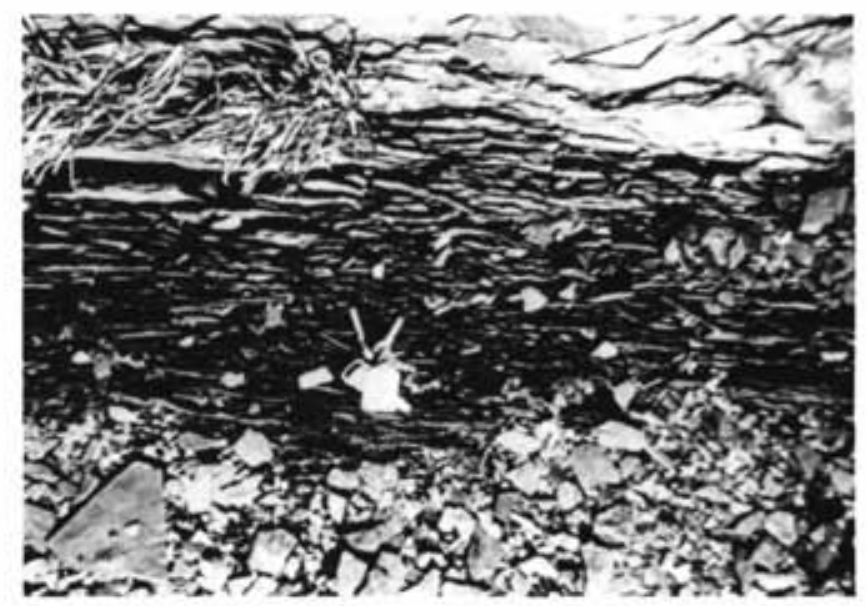

FIGURE 2. Cap du Caribou. Gelifraction d'une microfalaise de haut d'estran dans I'Ordovicien, et plaquettes de schistes tombees au pied. Cliche A. Guilcher, 1975.

Cap du Caribou. Frost shattering in a micro-cliff in Ordovician at the upper intertidal level; angular fragments of shales at foot of cliff.

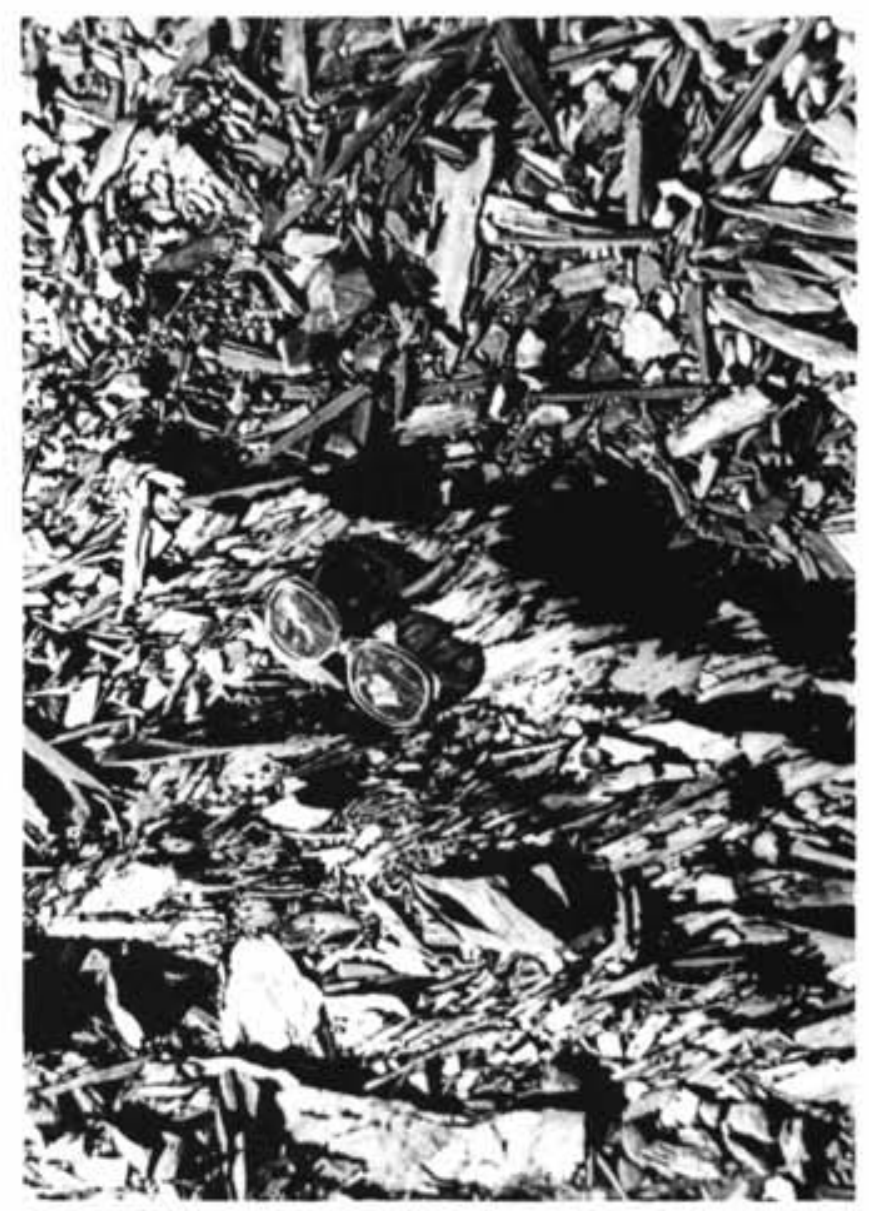

FIGURE 3. Pointe a la Cive. Gelifraction d'estran dans schistes ordoviciens (cliche A. Guilcher, 1973).

Pointe a la Cive. Gelifraction platform in Ordovician shales. 
gnant son maximum d'efficacité dans les parties supérieures de l'estran, et à la base des basses falaises lorsqu'il y en a. DIONNE (1972b), et TRENHAILE (1978) pour la côte nord de la Gaspésie, ont déjà noté cette localisation préférentielle altitudinale. On peut aussi l'observer vers le niveau moyen, mais guère plus bas. La gélifraction est favorisée par la multiplicité de petites mares sans profondeur (quelques centimètres seulement) dont l'eau gèle facilement à basse mer parce qu'elles n'ont aucune réserve thermique qui pourrait ralentir leur gel par convection, à l'inverse des eaux où la profondeur est de quelques mètres ou davantage. La gélifraction des schistes consiste en un émiettement en plaquettes totalement anguleuses. Ces plaquettes forment un remblaiement pelliculaire qui comble les dépressions entre les micro-arêtes structurales: plages de remblaiement et roche fournisseuse de débris alternent sur le même plan pour constituer un platier où les inégalités ne sont que décimétriques. Une partie des plaquettes est transportée par les vagues en haut d'estran, où elles constituent un cordon dont le sommet est au niveau des tempêtes, et où les plaquettes n'ont, le plus souvent, pratiquement pas encore été usées. Dans les meilleurs cas, il n'y a pas du tout de galets, ni sur le platier, ni dans le cordon: le matériel est resté anguleux, de sorte que le rôle de la dynamique marine est réduit à un transport à très courte distance. Le processus essentiel semble donc bien la cryoplanation. II arrive cependant qu'il y ait des galets de grès sur le platier en pourcentage plus ou moins grand. On peut alors penser que l'abrasion par les vagues joue un rôle certain à côté de la cryoplanation. A plusieurs endroits sur la rive sud existent des formes sculptées dues à l'abrasion littorale (DIONNE, 1961).

Les plates-formes ne se trouvant en général qu'à 2 ou $3 \mathrm{~m}$ en contre-bas de la terrasse Mic Mac ou Mitis

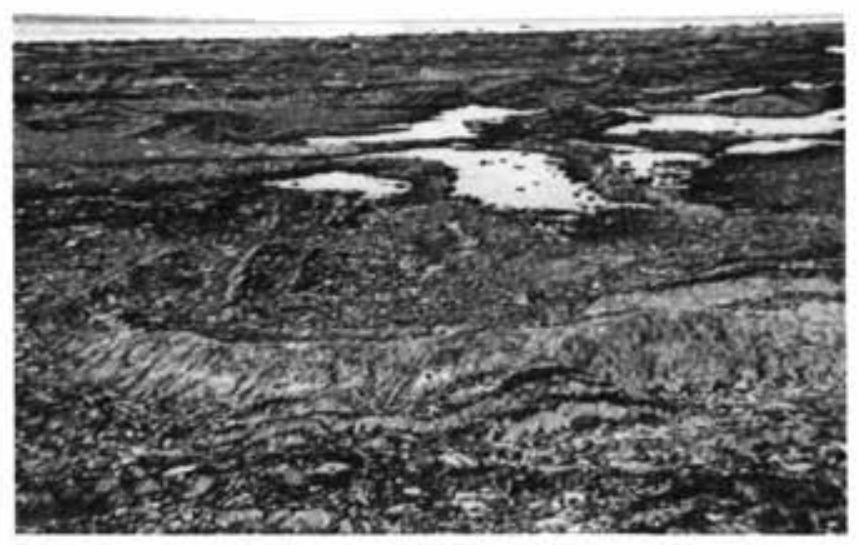

FIGURE 4. Pointe à la Cive. Cryoplanation d'estran dans schistes ordoviciens (cliche A. Guilcher, 1973).

Pointe a la Cive. Cryoplanation platform in Ordovician shales. (par exemple au cap du Caribou, à la pointe aux Cenelles: fig. 8 et 9 ), il est très vraisemblable qu'elles dérivent de sa partie externe par ablation d'une tranche mince de roche; la terrasse elle-même pouvant hériter de surfaces glaciaires ou pré-glaciaires. Héritage ne signifie évidemment pas conservation totale d'identité; là oủ il est le plus probable, c'est quand les plates-formes affectent le bas du versant nord-est d'une croupe appalachienne aplatie, simulant par leur forme un grand drumlin. Au demeurant, c'est en cette situation que se trouvent les plus vastes plates-formes, qu'il reste à caractériser individuellement: du SO au NE, celles de la pointe à la Cive, du promontoire de I'Orignal, du cap du Caribou, de la pointe aux Cenelles, et de la pointe Mitis (mais des plates-formes moins belles et à fonctionnement analogue existent en d'autres endroits, notamment à Sainte-Luce et à Sainte-Flavie). Le cap du Corbeau sera mentionné ensuite par contraste.

\section{LES SITES PRINCIPAUX}

a) Pointe à la Cive

L'estran de cryoplanation littorale de la pointe à la Cive (fig. 4 et 5), qui ferme à l'ouest l'anse à Mercier à Saint-Fabien-sur-Mer (DIONNE, 1979), mesure environ 250 sur $100 \mathrm{~m}$, l'estran total de cette pointe mesurant 500 sur $200 \mathrm{~m}$ environ. II constitue une plate-forme très régulière entre les hautes mers de grande marée et celles de morte eau. La cryoplanation y est évidente:
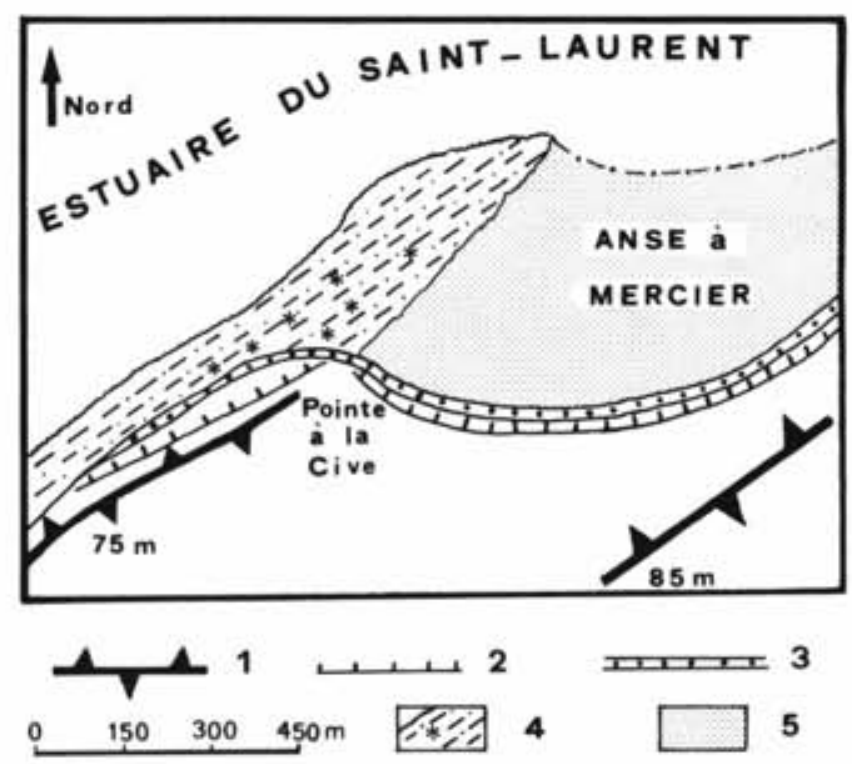

FIGURE 5. Pointe a la Cive. Légende des signes; 1) crete appalachienne ; 2) falaise Mitis (Mic Mac) ; 3) cordon littoral; 4) estran rocheux (etoiles: cryoplanation; points : elements détritiques); 5) estran vasosableux.

Pointe à la Cive. Captions: 1) Appalachian ridge; 2) Mitis (Mic Mac) cliff; 3) high water ridge; 4) rocky platform (stars; cryoplanation; dots: loose particles); 5) silt and sand. 
les plaquettes détacheees sont ultra-anguleuses. Au niveau des plus hautes mers, cependant, les bords des plaquettes ont subi un début de façonnement. Au même niveau et à celui des embruns, les schistes en affleurement gélivent aussi et assurent une fourniture d'élements.

Vers le bas, la plate-forme de cryoplanation passe progressivement, à partir du niveau des hautes mers de morte eau, à une plate-forme plus étendue de type moins zonal. Des galets apparaissent, fournis par les bancs gréseux intercalés dans les bancs schisteux. II $\mathrm{y}$ a tous les stades entre blocs gréseux anguleux, eux-mêmes détachés par gélifraction, et éléments arrondis. De petites plages de galets et éléments subémoussés se voient entre les micro-arêtes appalachiennes qui portent une couverture rase de Fucus.

En contournant la pointe en direction du SO, l'estran change de caractère. II est moins large et plus battu; il porte beaucoup de galets entre les micro-arêtes. Entre le niveau des mi-marees et celui des basses mers de morte eau apparaît une esquisse de cordon glaciel du type décrit plus loin. Ici, c'est donc plutôt un estran azonal dans sa partie haute et glaciel dans sa partie basse, alors qu'à la pointe la cryoplanation l'emporte dans la partie haute et l'érosion azonale dans la partie basse. La localisation de la cryoplanation prépondérante parait liée surtout au faciès.

\section{b) Promontoire de l'Orignal}

Le promontoire de l'Orignal (fig. 6 et 7) comprend deux plates-formes localisées sur la face orientale, c'est-à-dire du côté de l'anse à l'Orignal. La côte occidentale et le cap à l'Orignal lui-même sont beaucoup plus abrupts.

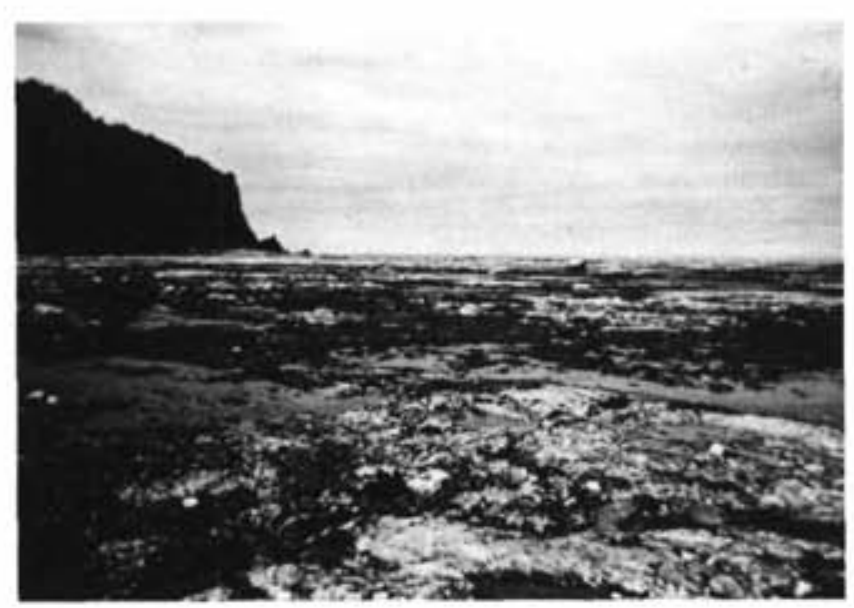

FIGUAE 6. Platier a l'est-nord-est du cap a rOrignal (le cap est au fond). Estran a cryoplanation dans schistes ordoviciens (cliche A. Guitcher, 1975).

Platform facing cap a rorignal in the East-South-East. Cape seen in the distance. Cryoplanation platform in Ordovician shales.
La plate-forme la plus au sud, située à la terminaison nord-est de la crête appalachienne nommée cap Rond, est petite, $70 \mathrm{~m}$ sur 35 , mais c'est peut-être la plus belle de toutes celles mentionnées ici pour la cryoplanation. La position altitudinale est la même: entre les plus hautes mers et les hautes mers de morte eau. Les micro-arêtes de schistes ordoviciens sont en voie d'aplanissement rapide, et sont séparées par de petites plages de plaquettes gélivées. Le processus est limité aux parties à faciès schisteux: lorsque le faciès devient gréseux et plus massif, la roche gélive peu ou point, et ces parties gréseuses forment des reliefs de 1,50 à $2 \mathrm{~m}$ de saillie au-dessus du reste. Plus bas, des galets apparaissent, remplaçant les plaquettes anguleuses: c'est donc bien une cryoplanation de haut d'estran.

La plate-forme la plus au nord, sur la face nord-est du promontoire de l'Orignal, est beaucoup plus vaste: 600 sur $400 \mathrm{~m}$, dont les 300 sur $200 \mathrm{~m}$ supérieurs peuvent être attribués à la cryoplanation. Cet estran est taillé dans des schistes gaufrés et plus ou moins plissotés, donc à pendage moins rigoureusement subvertical que d'ordinaire, avec des intercalations gréseuses minces. Le débitage est le même qu'ailleurs. Quelques galets bien roulés, parfois allogènes, sont mêlés aux plaquettes de cryoclastie; les allogènes peuvent être considérés comme d'apport glaciel. L'estran porte en outre quelques très gros blocs glaciels très apparents, I'un de conglomérat ordovicien, les autres précambriens et originaires de la côte nord. En arrière, le cordon de tempête à plaquettes schisteuses à angles vifs est très typique et de provenance cryoclastique évidente. II alterne avec de courtes falaises de 2 a $3 \mathrm{~m}$ de haut, où la même gélivation est très active: les petits bancs se détachent facilement à la main.

\section{c) Le cap du Caribou}

Au cap du Caribou (fig. 8), la plate-forme qui est accolée au nord mesure 750 sur $250 \mathrm{~m}$. Elle a un fonctionnement cryoclastique dans l'ensemble de sa superficie interne: micro-arêtes en voie de gélifraction, accumulation corrélative de plaquettes dans les creux, le tout aligné en bandes parallèles SO-NE dans la direction appalachienne. Le cap lui-même est un élément de la terrasse Mic Mac ou Mitis, splendide du cóté nord oủ la terrasse s'adosse à une falaise morte subverticale montant à 60-75 m. Un labour récent y montrait, en octobre 1980, une foule de plaquettes de schiste, indiquant que cette terrasse avait été élaborée, à 3-4 m au-dessus de l'actuelle, par les mêmes processus de cryoplanation que presentement.

Sur sa face sud, la terrasse s'abaisse vers l'estran actuel par un versant rocheux de 5 a $6^{\circ}$, passant sans discontinuité à une plage de même pente. Ce versant est une surface de cryoplanation inclinée, qui fournit des plaquettes à la plage associée: c'est une variante du 


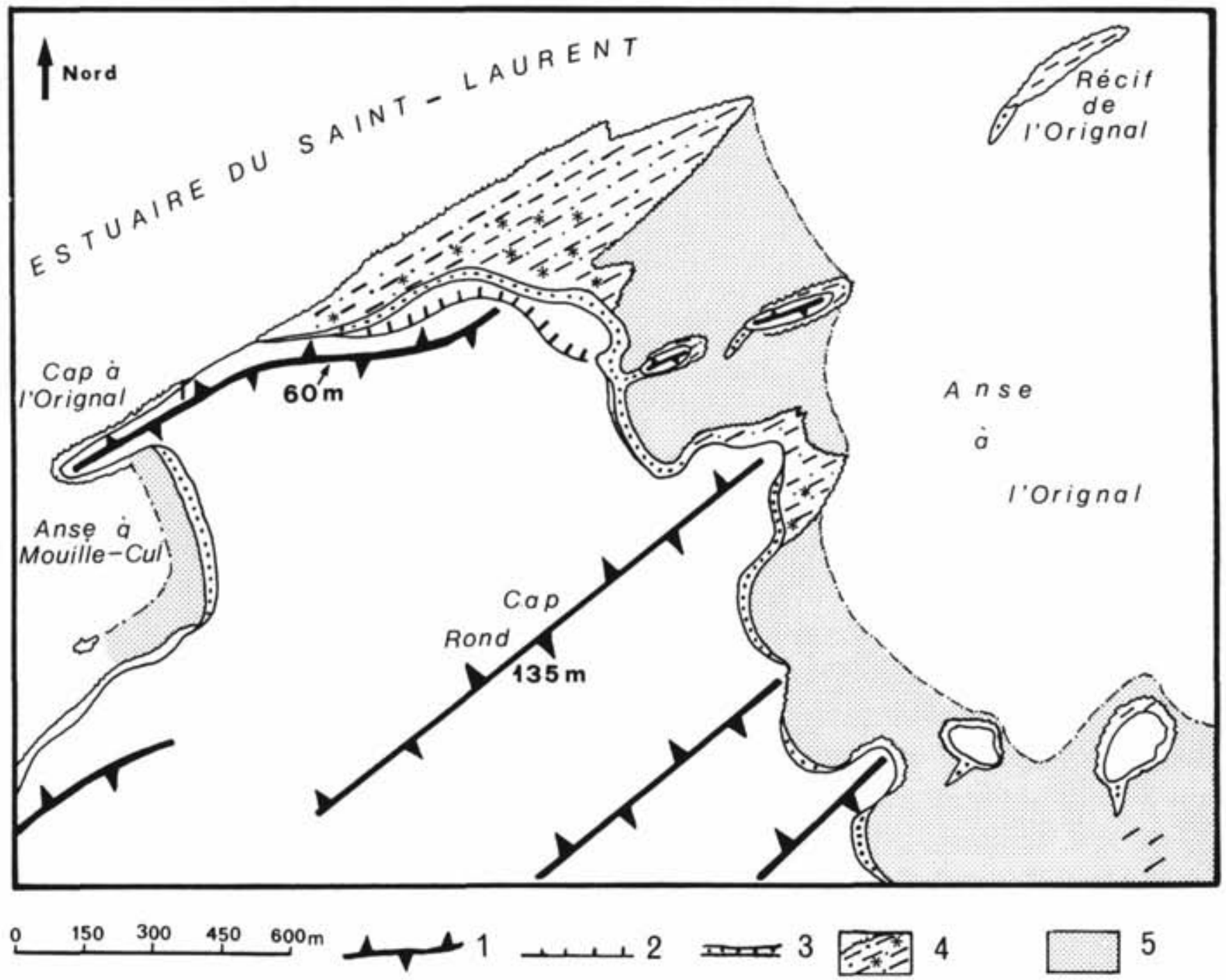

FIGURE 7. Promontoire de rOrignal. Légende des signes: 1) crête appalachienne : 2) falaise Mitis (Mic Mac) : 3) cordon littoral; 4) estran rocheux (etoiles: cryoplanation; points: elements detritiques); 5) estran sablo-vaseux.
Orignal headland. Captions: 1) Appalachian ridge; 2) Mitis (Mic Mac) cliff; 3) high water ridge; 4) rocky platform (stars: cryoplanation: dots: loose particles); 5) sand and silt. processus, avec comme différences la pente et l'extension beaucoup plus limitée.

\section{d) La pointe aux Cenelles}

La pointe aux Cenelles (fig. 9), située à $7 \mathrm{~km}$ au nordest de l'église de Sainte-Flavie, détermine comme les précédentes, du fait de la légère obliquité de la direction appalachienne avec la direction générale du littoral, un redan, avec report du littoral de $1700 \mathrm{~m}$ au sud dans la baie de Mitis. Entre la pointe et l'embouchure de la rivière Mitis, l'estran est sablo-vaseux; il est essentiellement rocheux sur la face nord-ouest de la pointe, longue de $1700 \mathrm{~m}$, et jusqu'à $800 \mathrm{~m}$ au nord-est de celle-ci.
Sur $900 \mathrm{~m}$ entre l'endroit oủ la route côtière quitte le rivage vers le sud et l'extrémité distale de la pointe, en avant d'une basse terrasse Mitis (Mic Mac) de $150 \mathrm{~m}$ de largeur, les rochers bas à micro-arêtes de très haut estran sont soumis à une fragmentation intense par gélifraction, fournissant une grande abondance de plaquettes schisteuses. A l'occasion d'un gel précoce de fin octobre 1980 , on a pu saisir cette gélifraction en cours sur le pourtour des mares. Les plaquettes détachées alimentent le cordon de tempête. Plus bas, comme d'habitude, la gélifraction cesse d'être prépondérante: dans les petits espaces détritiques interstitiels prédominent de petits galets de provenance glacielle. 


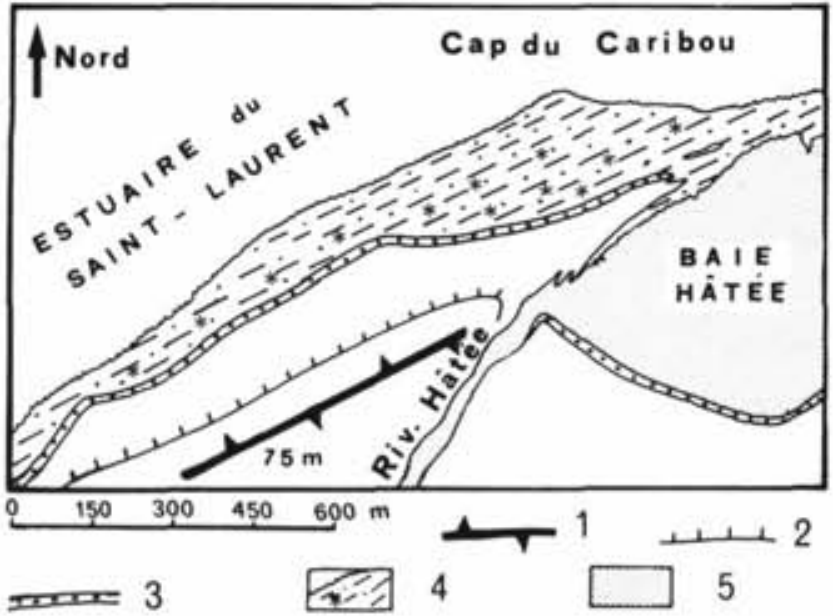

FIGURE 8. Cap du Caribou. Légende des signes: 1) crête appalachienne : 2) falaise Mitis (Mic Mac) ; 3) cordon littoral ; 4) estran rocheux (etolies: cryoplanation: points : elements détritiques) ; 5) estran sablovaseux.

Cap du Caribou. Captions: 1) Appalachian ridge ; 2) Mitis (Mic Mac) cliff: 3) high water ridge; 4) rocky platform (stars : cryoplanation; dots : loose particles); 5) sand and silt.

La pointe elle-même est prolongée par trois rochers allongés dans la direction appalachienne SO-NE, dont les têtes restent découvertes à basse mer. II s'avère que l'attaque principale de la houle vient du nord-est, car ces rochers comportent des queues de zone d'abri (queues de comète) détritiques du côté du sud-ouest. Ces queues sont constituées, pour les deux rochers les plus proches de la pointe, par des plaques et plaquettes de schistes et par du gravier anguleux, graduellement amenuisés en s'éloignant de la roche mère dans laquelle la fragmentation par gélifraction est parfaitement évidente. On ne peut pas voir mieux qu'ici les parts qui reviennent, et au processus cryergique fournisseur de débris, et à la dynamique marine qui répartit des débris sans toutefois avoir encore pu en façonner les éléments. Quant au troisième rocher, le plus éloigné vers le nord-est et décalé au sud par rapport au second, il a lui aussi une queue détritique orientée de même; mais les éléments de cette queue, de taille 2 à $10 \mathrm{~cm}$ pour ce qui n'est pas gravier, sont très sensiblement moins anguleux, certainement parce que ce site plus avancé est plus battu, ce qui contribue à préciser le rapport des forces en action.

\section{e) La pointe Mitis}

Nouvelle avancée basse en redan, longue de $800 \mathrm{~m}$ et sommée d'un phare, la pointe Mitis (fig. 10) est continuée sur 800 autres mètres par des crêtes rocheuses dont quelques-unes, notamment la plus avancée, sont hors de l'eau à haute mer de grande marée. La roche, dans la partie au nord-est du phare, est un grès assez compact et généralement peu diaclasé: aussi n'y a-t-il pas de phénomène de gélifraction importante en cette partie

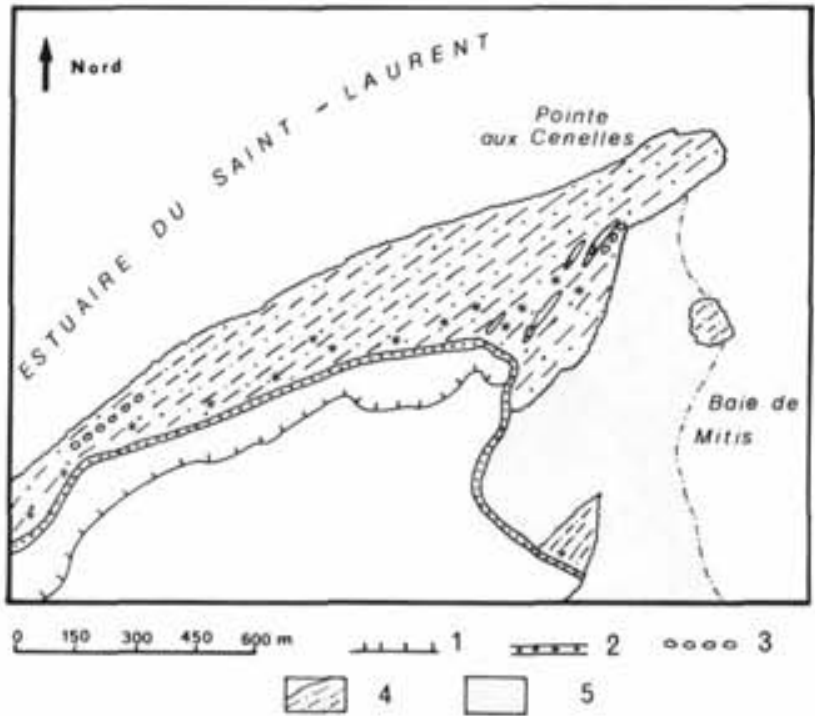

FIGURE 9. Pointe aux Cenelles. Légende des signes: 1) Falaise Mitis (Mic Mac) ; 2) cordon littoral: 3) cordon glaciel de basse mer; 4) estran rocheux (étoiles: cryoplanation: points : eléments détritiques). 5) estran sablo-vaseux.

Pointe aux Cenelles. Captions: 1) Mitis (Mic Mac) cliff; 2) high water ridge; 3) boulder barricade; 4) rocky platform (stars: cryoplanation; dots: loose particles); 5) sand and silt.

(les grès, d'une façon générale, peuvent aussi être gélifractés, mais nettement moins que les schistes). Entre les micro-arêtes s'observent de nombreuses petites grèves de galets, d'arrondi variable et parfois assez bon, mêlés à de très abondants débris coquilliers de lamellibranches (les bancs de moules foisonnent sur toute la côte considérée en cet article). Le tri par les vagues rassemble ces débris en petits amas blancs. Les parties déprimées sont occupées par un sable fin et un peu vaseux, avec mauvais classement.

A l'est et au sud du phare, il en va différemment. Là, le grès est plus fréquemment en plaquettes, et, dans l'étage des hautes mers de morte eau et de grande marée, les écaillements des bancs affleurant par la tranche sont frequents. La gélifraction y est efficace. De petits et moyens blocs peu ou point façonnés gisent dans les micro-dépressions intercalaires. II s'agit d'une gélifraction incipiente, et non d'une cryoplanation proprement dite, à l'inverse des estrans précédemment décrits.

Un fait qui situe la pointe Mitis par rapport aux autres hauts d'estrans plus gélifs est l'existence de deux formes de corrosion littorale toutes différentes. La première forme, sous le phare à l'est de lui, consiste en alvéolisations en nids d'abeille, de format 1 à $2 \mathrm{~cm}$, qui, comme les formes analogues décrites en bien des pays, sont au niveau des hautes mers. Ce type morphologique fréquent dans les grès apparaît donc ici dans un faciès de grain fin à bancs assez gros. La seconde forme, à 100-150 $\mathrm{m}$ au sud-ouest du point précédent, consiste 


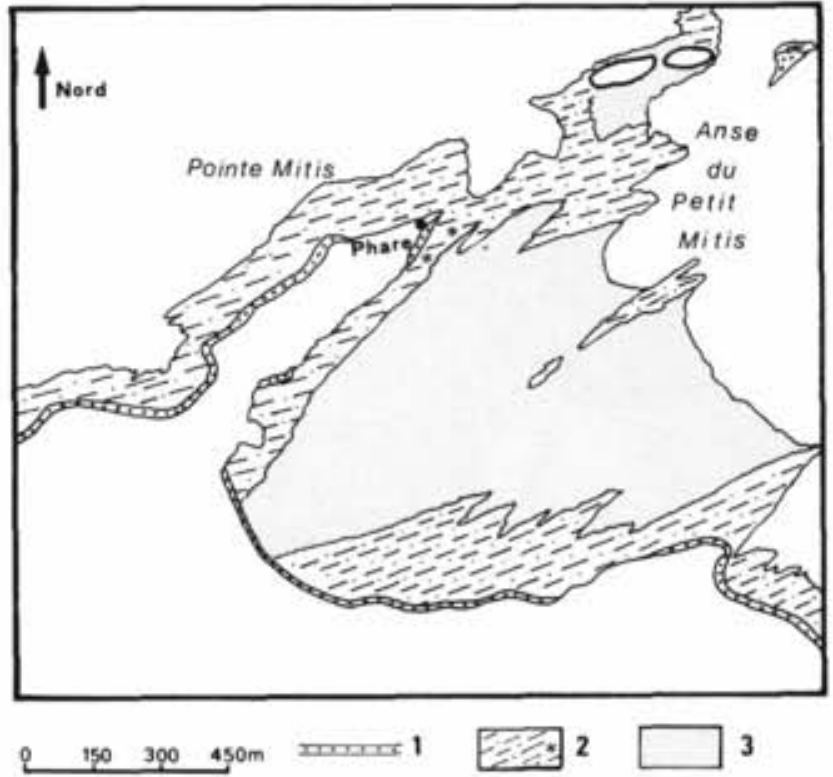

FIGURE 10. Pointe Mitis. Légende des signes: 1) cordon littoral; 2) estran rocheux (étoiles: gelifraction; points: eléments détritiques); 3) estran sablo-vaseux.

Pointe Mitis. Captions: 1) high water ridge; 2) rocky platform (stars frost shattering: dots: loose particles); 3) sand and silt.

en desquamations de grès mamelonnés ou plats, à pendage très incliné. Les écailles ont quelques millimètres d'épaisseur, et leur individualisation morphologique n'est pas due à la gélifraction.

\section{f) Le cap du Corbeau}

Ce site achève de préciser de façon antinomique les forces en action sur cette côte. Le cap du Corbeau (fig. 11) est formé, non pas de schistes gélifs, mais de grès et conglomérats calcaires massifs et très cohérents, qui affleurent dans des falaises de 20 a $30 \mathrm{~m}$ de hauteur bordées à leur pied par des méga-éboulis (DIONNE, 1962, p. 72; 1972c, p. 137). Ces roches, jointes à un certain nombre de petits ééments glaciels allogènes, fournissent dans les deux criques faisant suite à la pointe au nord-est de très abondants galets, fort bien roulés en ce site battu. Au lieu d'avoir ici des cordons de gélifracts point ou peu retouchés, on a affaire, par suite de la nature des roches, à des cordons de galets azonaux. Un affleurement très local de schistes sur le plancher d'une des criques est lui-même fortement abrase, parce que les galets sont constamment brassés sur sa surface.

Une autre forme non spécifique d'hivers froids est représentée aux abords immédiats de Rimouski, dans des conglomérats calcaires, par des vermiculations et des mares de corrosion à fonds plats et encorbellements périphériques (DIONNE, 1967), analogues à celles qu'on peut observer dans les calcaires littoraux du mas-

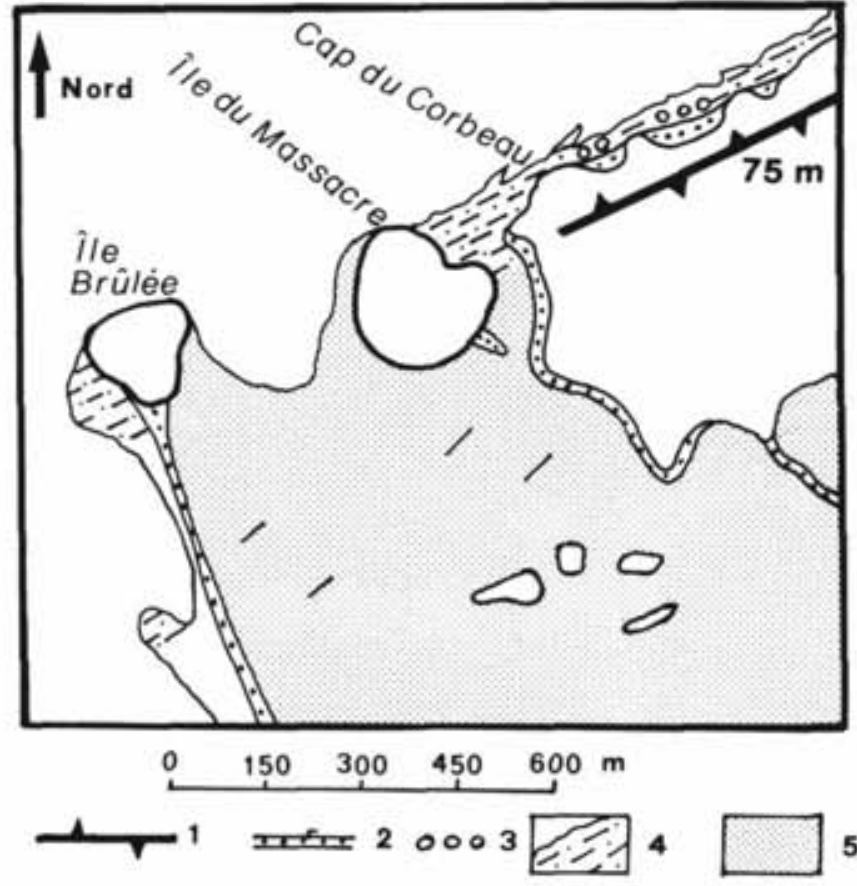

FIGURE 11. Cap du Corbeau. Légendes signes: 1) crête appalachienne; 2) cordon littoral et queve en position d'abri; 3) cordon glaciel de basse mer; 4) estran rocheux; 5 ) estran sablo-vaseux.

Cap du Corbeau. Captions: 1) Appalachian ridge; 2) high water ridge and leeward spit: 3) boulder barricade; 4) rocky platform; 5) sand and silt.

sif Armoricain et des iles britanniques, sous climat à hiver doux (GUILCHER, 1952, et 1954, p. 47 et pl. IB).

Vermiculations et mares à encorbellements dans les calcaires, alvéolisations en nids d'abeille et desquamations dans les grès, grèves de galets azonales dans les roches massives, voilà autant de faits qui montrent que la gélifraction littorale n'est pas universelle sur la côte sud du Saint-Laurent. Pour qu'elle s'exer$\mathrm{Ce}$, il faut que la roche s'y prête. C'est une vérité de La Palisse, mais il faut l'énoncer.

\section{TERMES PROCHES DE COMPARAISON}

La gélifraction littorale a également été mentionnée à la pointe d'Argentenay, ile d'Orléans près de Québec (CHAMPAGNE, 1978, p. 54-55). Cet auteur note la différence des gélifracts selon que la roche est schisteuse ou gréseuse. Ses observations en la matière concordent avec les nôtres.

Un autre terme de comparaison est le banc de SaintVallier sur la rive sud de l'estuaire du Saint-Laurent en face de l'île d'Orléans, que nous avons visité à très grande basse mer en octobre 1980 en compagnie de Jean-Claude Dionne et de Michel Allard. La plate-forme littorale, formée de schistes ordoviciens subverticaux, tantôt en lames minces, tantôt en bancs d'épaisseur 
d'environ $5 \mathrm{~cm}$, mesure approximativement $1000 \mathrm{~m}$ de long. Son étagement est le même que dans la région de Rimouski: cordon de très haute mer de plaquettes de schistes non ou très peu usées: puis, plus bas, plateforme rocheuse très régulière, avec seulement des micro-arêtes de $1 \mathrm{~m}$ de haut au maximum, et forte gélifraction jusqu'au niveau de mi-marée, qui fournit les élements du cordon surincombant, et ceux de petites plages de remblaiement des légers creux de la plateforme. En sa partie la plus basse, le platier est couvert d'une mince pellicule de vase. Selon Dionne (verbatim. 1980), cette pellicule n'existe pas toujours. II s'agit donc encore ici d'une plate-forme de cryoplanation en sa partie haute, qui porte en outre des méga-blocs glaciels dispersés. Comme au cap du Caribou, à la pointe aux Cenelles et ailleurs, le banc de Saint-Vallier constitue la terminaison nord-est d'une crête appalachienne.

Au cours de la même visite, nous avons vu à l'embouchure de la rivière Boyer et à l'enracinement du banc de Saint-Vallier sur la route de Saint-Michel, deux coupes de plages holocènes isostatiquement soulevées, appartenant à la mer de Goldthwait et à sa récession. Ces plages sont constituées de plaquettes schisteuses très anguleuses, qui montrent une genèse par gélifraction analogue à celle observée de nos jours : ce qui est à rapprocher de nos observations sur la terrasse du cap du Caribou.

En un article récent sur la côte nord-est de la presqu'ile de Gaspé, TRENHAILE (1978, p. 98-99) a remarqué que « l'érosion mécanique par le gel et la glace côtière doit être considéréen, que * les effets de l'action du gel sont manifestes $\%$, et particulièrement efficaces audessus du niveau moyen (nous avons nous-même noté ce dernier point, on l'a vu, sur la cóte de Rimouski). Trenhaile parait cependant considérer ces actions comme relativement accessoires en Gaspésie : il s'attache plus particulièrement aux processus d'abrasion, et apporte d'intéressantes considérations dans la ligne de l'école australasienne des plates-formes littorales, illustrée par BARTRUM (1926), EDWARDS (1951), HILLS (1949) et autres. Plus récemment, TRENHAILE et RUDAKAS (1981) donnent les résultats d'expériences de gélifraction qu'ils ont effectuees sur des roches de la côte nord de Gaspésie. Ils concluent que les roches les plus gélives sont les schistes (ce qui est certain en tout état de cause); que la gélifraction maximale se réalise avec des eaux saumâtres et non en celles de salinité « normalen ; et que, si les processus de gélifraction sont incontestablement efficaces, il n'y a cependant pas de preuve qu'ils créent véritablement des plates-formes littorales subhorizontales en Gaspésie.

Ces conclusions méritent beaucoup d'attention. II faut cependant noter au préalable que les très nombreuses expériences de cryoclastie effectuées au Centre de géomorphologie de Caen, notamment dans les schis- tes (LAUTRIDOU et al., 1971, p. 48, 49, 55), montrent la nécessité de nombreux cycles de gel-dégel pour arriver a une efficacité (entre 160 et 350 selon les expériences), ce qui résulte aussi d'observations faites à Sakhaline (GUILCHER, 1980); que les expériences de TRENHAILE et RUDAKAS (1981) semblent avoir comporté un nombre beaucoup plus limité de ces cycles, et que, de ce fait, les résultats qu'ils ont néanmoins obtenus portent à conclure à une rapidité relativement très grande dans les schistes ordoviciens de Gaspésie.

Par ailleurs, le rôle de la salinité semble très naturel. On peut penser qu'il s'accorde avec nos propres observations, selon lesquelles c'est sur les hauts d'estrans que la gélifraction est maximale. Au reste, la salinité est plus faible à Rimouski qu'en Gaspésie.

Quant à la possibilité de véritable réalisation de plates-formes de cryoplanation littorale, nous ne pensons pas nous non plus que la gélifraction actuelle ait créé ex nihilo les plates-formes des bords du SaintLaurent, qu'elles soient situées en Gaspésie, à l'île d'Orléans, à Saint-Vallier ou dans la région de Rimouski. La cryoplanation actuelle a donné à Saint-Vallier une empreinte et un façonnement terminaux sur la base des crêtes appalachiennes dont l'histoire a dû être longue et complexe (Dionne et Allard, verbatim, 1980). Et plus généralement, l'idée d'héritages pour l'ensemble des plates-formes rocheuses bordant l'estuaire du Saint-Laurent a été exprimée (DIONNE, 1972b). Nous pensons toutefois que l'empreinte terminale cryoclastique est essentielle sur les estrans schisteux de la région de Rimouski au-dessus du niveau moyen, et suffisante pour maintenir l'expression de plate-forme de cryoplanation. A notre avis, cette empreinte serait peut-être supérieure à ce que pensent TRENHAILE et RUDAKAS (1981) pour la Gaspésie, dont nous avons examiné la côte en 1973, à l'est de Sainte-Anne-des-Monts.

\section{CORDONS GLACIELS DE BASSE MER (BOULDER BARRICADES)}

\section{LES FAITS D'ENSEMBLE DANS LA RÉGION DE RIMOUSKI}

Sur la partie externe de nombreux estrans de la région de Rimouski s'observent des accumulations de gros blocs en alignements parallèles à la côte, dont les éléments, généralement arrondis, comprennent une proportion considérable de roches de Précambrien : ils proviennent donc surtout de la cóte nord, ainsi que les blocs glaciels qui existent sur les estrans en ordre beaucoup plus dispersé (DIONNE, 1972c).

\section{a) Site du Sacré-Coeur-de-Rimouski}

Le dispositif peut être illustré par la partie sud de la grève du Sacré-Coeur-de-Rimouski (fig. 12, 13, 14, 15, 

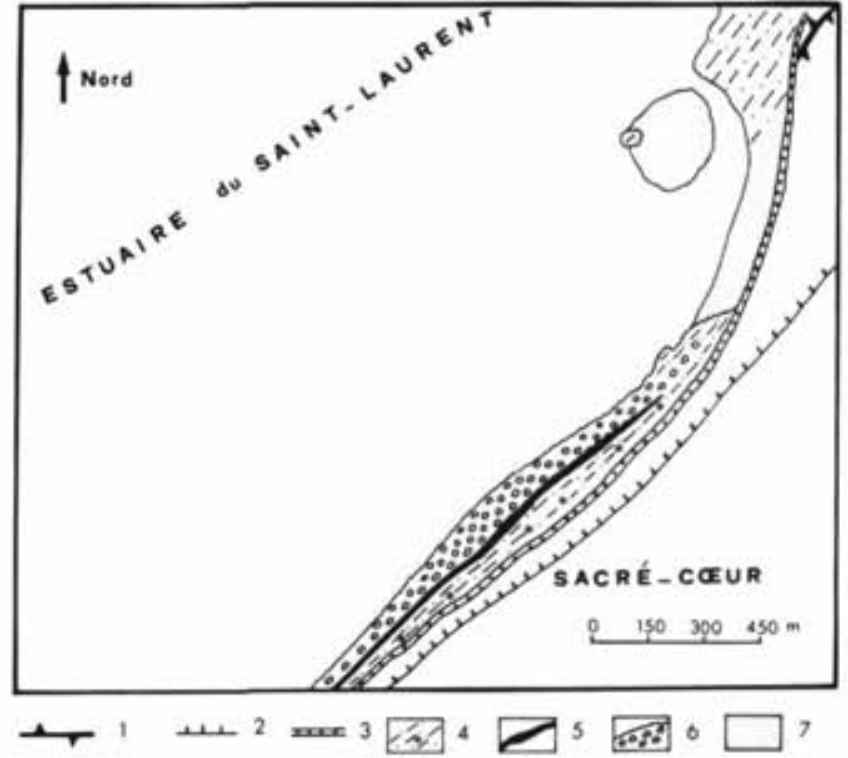

FIGURE 12. Sacré-Coeur de Rimouski. Carte de r'étagement. Légende des signes : 1) crète appalachienne ; 2) talaise Mitis (Mic Mac) et terrasse a son pied; 3) cordon littoral de haute mer; 4) estran rocheux de cryoplanation (étoiles: cryoplanation: points : éléments détritiques) ; 5) mare longitudinale barrée: 6) cordon glaciel de basse mer; 7 ) estran sablovaseux.

Sacre-Cour de Rimouski. Captions: 1) Appalachian ridge; 2) Mitis (Mic Mac) cliff and terrace at foot of cliff; 3) high water ridge; 4) cryoplanation platform (stars: cryoplanation; dots: loose particles). 5) longitudinal dammed pond; 6) boulder barricade; 7) sand and sitt.
16), que nous considérons comme le locus tipicus pour la région de Rimouski, et qui s'étend sur au moins $3 \mathrm{~km}$ au-delà vers le sud-ouest. De haut en bas, on observe:

- La falaise Mitis ou Mic Mac, avec sa raideur habituelle, taillée dans la roche en place, et dont la base est à environ $8 \mathrm{~m}$ au-dessus des hautes mers actuelles.

- La terrasse Mitis ou Mic Mac, plus fortement inclinée que d'ordinaire, qui descend jusqu'à $1 \mathrm{~m}$ au-dessus des plus hautes mers actuelles. Elle porte la voie ferrée du Canadien National, et, au bord de la mer, des chalets. Elle est formée en surface de gravier marin sur une épaisseur vraisemblablement faible (1 à $2 \mathrm{~m}$ ).

- Le cordon de tempête et de très hautes mers, et les rochers associés. Le cordon est formé, comme d'ordinaire sur cette côte, de gravier et plaquettes de schistes dérivant par gélifraction de la roche avoisinante shistogréseuse. La gélifraction est activement en cours dans la roche entre le niveau des plus hautes mers et celui des hautes mers de morte eau. Elle commence dès fin octobre-début novembre autour des mares. Les plaquettes du cordon ont subi une certaine usure, ce qui indique une contribution de la dynamique marine. En-dessous du niveau des hautes mers de morte eau, la plate-forme rocheuse est aplanie dans l'ensemble et rugueuse dans le détail, et de véritables galets apparaissent, mêlés à des plaquettes gélifractées, en pourcentage variable selon les endroits; elle provient de l'ablation par gélifraction survenue à un niveau immédiatement au-dessus, et continue d'être usée aujourd hui par abrasion.

- Une mare longitudinale barrée, un peu au-dessus de la basse mer de morte eau, de profondeur 2 à $10 \mathrm{~cm}$,

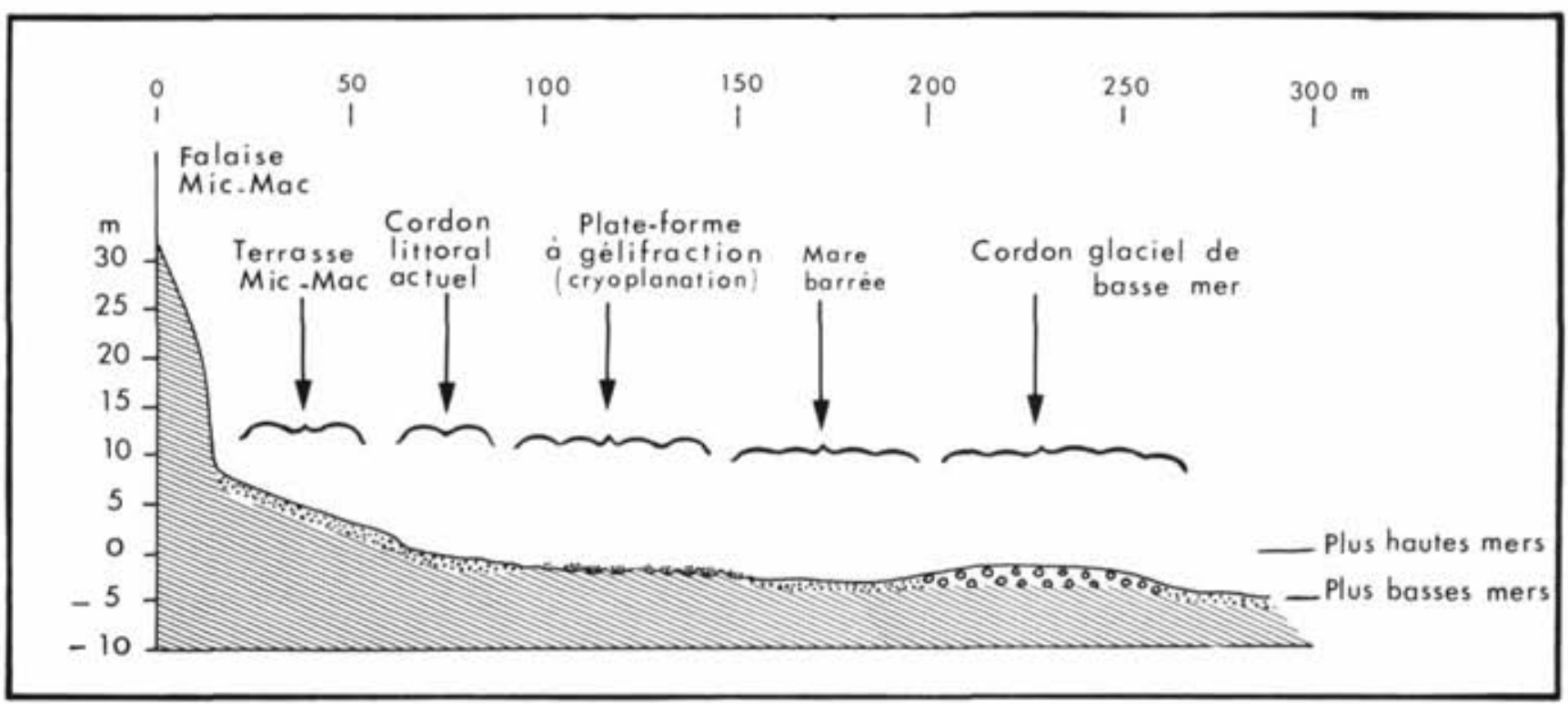



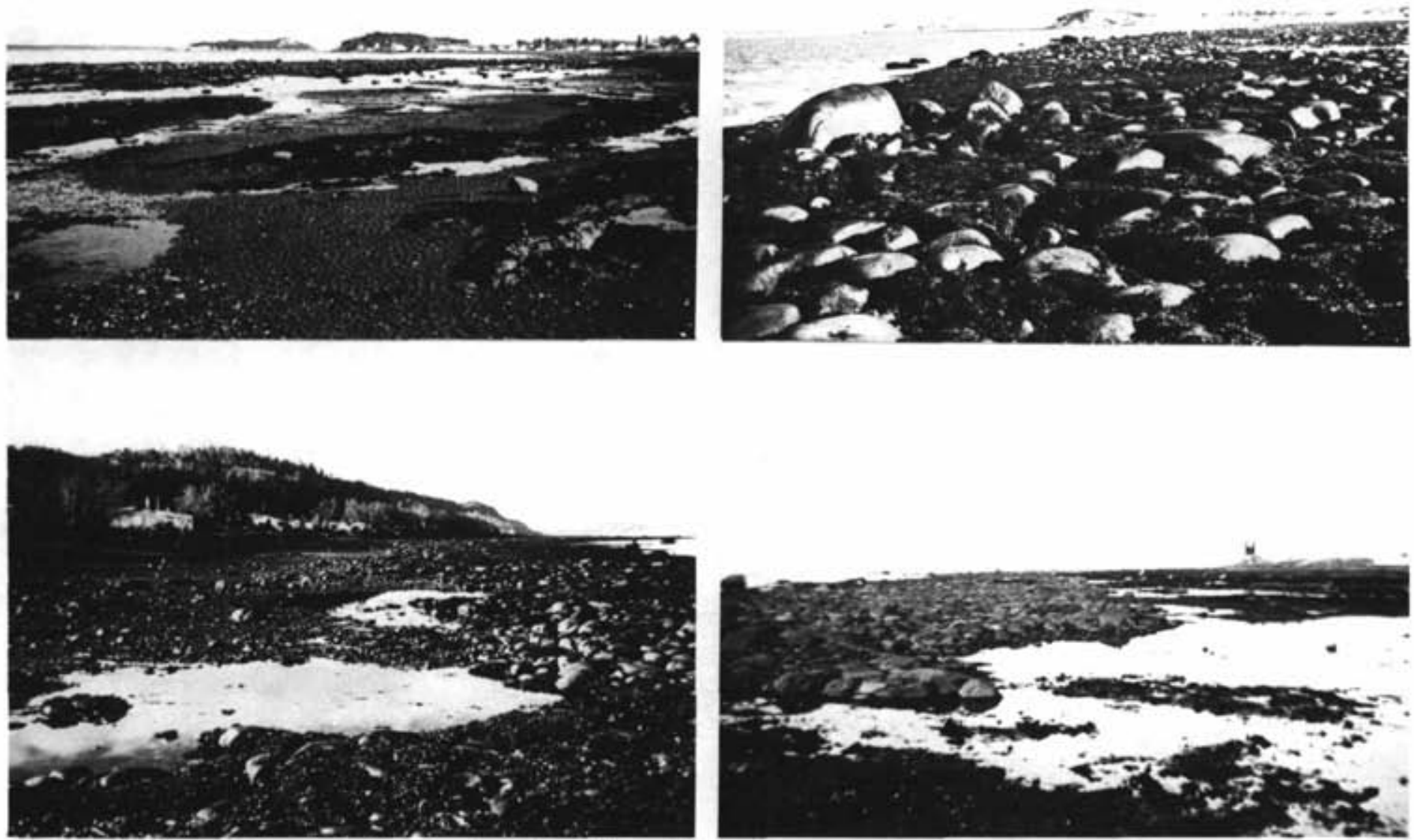

FIGURE 14. Sacre-Coour de Rimouski. Vue d'ensemble de l'estran (ct. figs. 12 et 13). Cordon glaciel de basse mer à l'arrière-plan (cliche $A$ Guilcher, 1975).

Sacre-Caur de Rimouski. General view of shore (ct. figs. 12 and 13). Boulder barricade in the distance.

FIGURE 15. Sacre-Conur de Rimouski. A droite: cordon glaciel de basse mer. Au centre : mare barrée. A gauche et en arriere : cordon de haut d'estran, terrasse et falaise Mic Mac (Mitis) (cliche A. Guilcher, 1975).

Sacre-Caur de Rimouski. Right: boulder barricade. Middle: dammed

avec une végétation de Zostères (en 1980). Elle est tapissée de sable fin et limon (silt) constituant une pellicule sur la roche.

- Un cordon de gros blocs, très continu comme la mare qu'il barre, formé d'éléments allogènes (cristallins) arrondis, de longueur moyenne supérieure à $0,40 \mathrm{~m}$, atteignant parfois plus de $1 \mathrm{~m}$. Les très gros blocs de un mètre cube ou davantage sont rares, et localisés sur le bord externe du cordon. Ils sont très serrés les uns contre les autres, avec parfois de beaux dispositifs de dallages; une végétation abondante de Fucus et d'Asco-

pond. Left and distance: high water ridge, Mic Mac (Mitis) terrace and clitt.

FIGURE 16. Sacre-Coour de Rimouski. Détail du cordon glaciel de basse mer (cliche A, Guilcher, 1980).

Sacre-Cœur de Rimouski. Closer view of boulder barricade.

FIGURE 17. Entre Sainte-Flavie et Sainte-Luce. Cordon glaciel de basse mer et mare barrée (cliché A. Guilcher, 1980).

Between Sainte-Flavie and Sainte-Luce, Boulder barricade and dammed pond.

phyllum pousse dans les interstices étroits. Le sommet de ce cordon est entre le niveau moyen et celui de la haute mer de morte eau. Le pied externe est au niveau des grandes marées basses: le cordon finit là sur une plaine à gravier, petits galets en pavage, et quelques blocs glaciels épars. Des Laminaires ont fixé leurs crampons sur une partie des blocs, et poussent en ordre dispersé sans former un revêtement (les Laminaires sont abondants sur toute la grève du Sacré-Coeur). Dans sa partie nord-est, le cordon glaciel finit vers le bas sur une plaine de sable émergeant même en morte eau sans rupture de pente. 
b) Autres cordons glaciels de basse mer aux environs de Rimouski

Ces cordons, nombreux au total, sont caractérisés ciaprès du nord-est au sud-ouest (fig. 1).

Dans l'anse des Morts (partie nord-est de la baie de Mitis), un cordon glaciel du type Sacré-Coeur existe de façon très continue, avec mares en arrière entrecoupées de micro-arêtes de grès schisteux à gélifraction modérée. Le haut d'estran et la succession des formes sont fondamentalement les mêmes qu'au Sacré-Coeur. Les blocs glaciels ont une longueur généralement inférieure à $0,50 \mathrm{~m}$, mais ils forment véritablement un cordon de 15 à $20 \mathrm{~m}$ de large, jusqu'au niveau des basses mers de morte eau. En outre, quelques grands blocs glaciels sont épars sur l'estran, comme à l'ordinaire.

Sur la face ouest de la pointe aux Cenelles (fig. 9), à environ $250 \mathrm{~m}$ à l'est de l'endroit oủ la route côtière quitte le rivage, un cordon glaciel bien caractérisé repose sur le platier rocheux entre le niveau des basses mers de morte eau et celui des basses mers de marée moyenne. Au-delà vers le bas, le platier rocheux se poursuit sans rupture de pente.

Au sud-ouest du môle de Sainte-Flavie (fig. 17), un autre cordon glaciel se trouve dans la même position que le précédent, c'est-à-dire au même niveau et avec banc de sable sans rupture de pente au-delà du cordon jusqu'au niveau des basses mers de grande marée.

A $4 \mathrm{~km}$ au sud-ouest du précédent, un autre élément est visible, avec une largeur d'environ $50 \mathrm{~m}$, et le même dispositif. De même à la pointe aux Coques (SainteLuce), avec mare barrée.

Plus généralement, les cordons glaciels sont fréquents entre Sainte-Luce et la pointe Mitis. Ils tendent cependant à manquer en deux sites: les anses sablovaseuses où le sédiment fin affleure jusqu'au bas de l'estran, comme à l'anse aux Coques (Sainte-Luce) et à la baie de Mitis; et les pointes à cryoplanation littorale étendue, comme l'extrémité distale de la pointe aux Cenelles (fig. 9).

Au cap du Caribou, face nord, un cordon glaciel s'esquisse sur le bas de l'estran décrit plus haut pour sa cryoplanation, dans un ensellement qui a dû favoriser les échouages de glaçons de dérive. On l'observe sur quelques dizaines de mètres de long; vu ses caractères imparfaits, il n'est pas porté sur la figure 8.

Enfin, au cap du Corbeau (fig. 11), les deux criques à cordons de galets roulés de haute mer qui se trouvent immédiatement au nord-est du cap portent elles aussi, à un niveau plus bas, des cordons glaciels de basse mer. Le plus typique est celui de la seconde crique, qui est séparé du bas du cordon de galets de haute mer par une petite mare type Sacré-Coeur.

Ces observations ne sont pas limitatives. L'estran n'ayant pas été vu en détail de bout en bout de la pointe Mitis jusqu'à la pointe à la Cive, il est très vraisemblable que des cordons glaciels de basse mer existent ailleurs dans la région de Rimouski.

\section{LES BOULDER BARRICADES DE RÉFERENCE}

En fait, ce type d'accumulations a déjà été décrit sur d'autres rivages à gel hivernal de l'Amérique du NordEst. II a été nommé boulder barricade par les auteurs de langue anglaise (DALY, 1902).

DIONNE (1962, p. 71) a déjà énuméré plusieurs des ensembles décrits ci-dessus, et a figuré celui de TroisPistoles. D'autres mentions et figures du même se trouvent dans 1972c, p. 128, 129, 134, 135.

BROCHU (1961) en a mentionnés en amont de Québec sur le Saint-Laurent, entre le pont de Québec et Portneuf. II a observé que ces cordons ne sont jamais situés dans les parties hautes ni même moyennes de l'estran: mais que, dans les parties basses, il en existe parfois deux et même trois alignements parallèles (ce que nous n'avons pas vu autour de Rimouski), ces multiplications étant associées aux estrans les plus larges et par conséquent les moins inclinés. Pour lui, le processus est le suivant: les blocs sont initialement déposés par les glaces de dérive "au hasard de l'échouement des glaces; puis, aux marées suivantes, les glaces agissant comme de puissants bulldozers peuvent repousser les blocaux abandonnés sur les estrans, mais, suivant la loi de la gravité, ces déplacements se produisent en direction de la partie inférieure de l'estran n.

D'autre part, un article très récent de ROSEN (1979) donne une description détaillée de cordons de blocs du même type dans une baie du Labrador, à Makkovik. Cet article contient un historique du problème et une explication génétique, dont nous donnons un résumé, avant de discuter nous-même l'origine à la lumière des sites de la région de Rimouski et d'observations qui y ont été faites en hiver et au printemps.

De tels cordons ont été décrits et figurés depuis fort longtemps par LYELL (trad. fr., 1873, vol. 1, p. 507) d'après des observations au Labrador et en mer Baltique. C'est DALY (1902) qui a proposé pour eux le nom de boulder barricades, à propos d'études au Labrador. TANNER (1939) a aussi apporté une contribution sur des cordons légèrement différents, de même que BIRD (1964) et LAURIOL et GRAY (1980) pour l'Arctique canadien. Tout le monde est d'accord pour attribuer à des processus glaciels ces cordons, qui sont de toute évidence un phénomène de régions oủ la mer gèle régulièrement et longuement. Une mise en place par les vagues seules est exclue, vu la localisation en bas d'estran, la dimension des éléments et l'absence de houles océaniques. Mais il reste à savoir de quel (ou quels) processus il s'agit exactement, car les transports glaciels "ordinaires" n'aboutissent qu'à déposer des 
blocs allogènes au hasard des échouages, sans former de "barricades " continues.

\section{DISCUSSION DES EXPLICATIONS ANTÉRIEURES}

Nous hésitons à accepter l'explication de BROCHU (1961). Sur des estrans dont la pente est généralement très faible, on voit mal comment des " forces de gravité " peuvent amasser les blocs en position basse. De plus, si les glaces flottantes agissent comme de "puissants bulldozers n, ce doit être non pas pour faire descendre les blocs, mais plutôt, ou même essentiellement, pour les remonter sous la poussée de la houle par tempête soufflant vers la terre, en constituant un classique ice wall bien connu par les descriptions et figurations qu'on en a faites en mer Baltique, et notamment en golfe de Riga (SLAUCITAJS, 1929). On revient alors, d'ailleurs, à des accumulations de haut d'estran, ce qui n'est pas le cas.

Pour ROSEN (1979), les choses se passent au Labrador central de la façon suivante. La débâcle se produit en moyenne à la mi-mai. Les premières eaux libres apparaissent à l'embouchure des cours d'eau (au Québec laurentien aussi). "Après la fusion initiale du couvert neigeux à la surface de la couche de glace dans la zone intertidale, des couches de vase du fond apparaissent [entre le cordon de blocs glaciels et le haut de l'estran]. La couleur foncée de la vase diminue l'albédo de telle sorte qu'une plus grande quantité de chaleur est absorbée, ce qui accroit subséquemment la fusion de la glacen. Cette fusion survient donc en arrière de la boulder barricade, et la localisation de celle-ci n'est pas attribuée à des échouages de floes chargés de blocs contre un pied de glace, puisque ce pied de glace fond précocement. La boulder barricade se forme donc là où il y a rupture de pente au niveau de la basse mer dans la plate-forme littorale. Les glaçons s'échouent parce que leur tirant d'eau les empéche d'aller plus loin; puis, lorsque des blocs ont commencé à se déposer, un effet d'accumulation se produit. Rosen croit que dans l'estuaire du Saint-Laurent les boulder barricades sont rares parce qu'on n'y trouve pas ou guere cette rupture de pente au niveau de la basse mer; il pense pouvoir se fonder sur les publications de Dionne pour dire que l'estuaire du Saint-Laurent est plutôt favorable à des échouages de blocs dispersés, à des dallages, etc., qu'à des cordons glaciels de basse mer.

En fait, à l'inverse de ce qu'écrit ROSEN (1979), les cordons glaciels de basse mer sont nombreux le long du Saint-Laurent (BROCHU, 1961; DIONNE, 1962, 1972c; et nous-même ici). Et l'explication par rupture de pente de bas d'estran n'est pas généralisable au SaintLaurent, puisque les cordons glaciels de basse mer s'y rencontrent indifféremment en des endroits où la plateforme ne découvre à grande marée basse que jusqu'à la base du cordon (cas du Sacré-Cœur pour l'ensemble du cordon), et en des endroits où le cordon s'est dépo- sé sur une plate-forme sans rupture de pente topographique, avec un découvert assez large au-delà du cordon vers le bas (cas à la partie nord-est du cordon du Sacré-Cœur, sur la face nord de la pointe aux Cenelles. et au sud-ouest du môle de Sainte-Falvie). Nous en concluons que l'explication proposée par Rosen ne s'applique pas à la région de Rimouski, ni non plus. pour les mêmes raisons, en amont de Québec.

\section{EXPLICATION PROPOSEE POUR LA REGION DE RIMOUSKI}

L'explication ci-après est proposée à la suite d'échanges de vues avec des collègues et un étudiant québécois qui ont fait de nombreuses observations pendant plusieurs années au moment de la débâcle, période de dépôt des blocs par les glaces flottantes.

Tous leurs témoignages, descriptions et photographies, concordent pour conclure que, lors du dégel, it subsiste fréquemment un épais et haut pied de glace côtier qui scelle le haut de l'estran, alors que la partie immédiatement en avant est déjà dégelée et que des glaçons peuvent y circuler sur la mer libre. Ainsi, lors de la débâcle de 1979, les observations de Rouillard (verbatim), avec photographies à l'appui, ont montré que, dans la seconde crique du cap du Corbeau (fig. 11), le pied de glace formait un mur vertical de plus de $1 \mathrm{~m}$, recouvrant totalement et solidement l'estran de haute mer, en arrière du bas de l'estran déjà libre de glace, et sur lequel se voyait un cordon glaciel déposé antérieurement. Dans la mesure où les glaces de dérive incluent des blocs rocheux, elles les déposent donc, lors de la fusion, en avant du reliquat de pied de glace qui interdit l'accès du haut de l'estran, et qui est, par son origine locale, le contraire d'un ice wall accumulé par une tempête (SLAUCITAJS, 1929). Telle est donc l'origine des cordons glaciels de niveau de basse mer sur la rive sud de l'estuaire du Saint-Laurent.

De toute évidence, le bel arrondi qui caractérise les blocs glaciels de tous les cordons de basse mer (cf. figs. $15,16,17)$ n'a pas été acquis sur leurs gisements actuels, mais est antérieur à leur transport par glaces flottantes. II a dô être réalisé au sein des formations fluvio-glaciaires si abondantes sur la cóte nord de l'estuaire du Saint-Laurent, source essentielle de ces matériaux (DIONNE, 1972c, p. 130-131).

Bien entendu, nous ne contestons pas l'explication de ROSEN (1979) pour le Labrador central, que nous n'avons pas visité. Nous disons seulement, avec l'accord des observateurs cités (ct. infra, remerciements), que sur le Saint-Laurent c'est l'explication proposée ci-dessus qui est valable.

II semble préférable de rester réservé sur l'exacte insertion, dans le type "cordons glaciels de basse mer" ou "boulder barricades", des cordons de blocs que 
LAURIOL et GRAY (1980) ont observés dans la baie d'Ungava (Québec arctique). Ces accumulations ressemblent, certes, en une large mesure à celles du Saint-Laurent; elles sont toutefois nettement plus volumineuses et plus hautes, et leurs conditions de gisement ne sont pas les mêmes. L'explication proposée diffère de la nôtre, mais, vu les différences de structure, on peut penser qu'il existe plus d'un sous-type de cordons glaciels de base mer, dont les mises en place ne seraient pas toutes semblables à celles de la région étudiée ici. De nouvelles études comparatives, portant sur des sites divers, seraient très souhaitables. Elles pourraient mettre mieux en lumière la variété et la complexité des actions glacielles.

Pour s'en tenir au Saint-Laurent, un point qui reste à éclaircir est la présence de deux ou trois cordons parallèles en certains endroits en amont de Québec. BROCHU lui-même (1961), qui les signale, n'est pas très catégorique à ce sujet. Une explication qu'on peut émettre, et à laquelle souscrit Dionne (in litteris), est que, selon les années, le pied de glace résiduel lors du dégel peut être plus ou moins large, de sorte que le dépôt de blocs en cordon peut se faire plus ou moins loin sur les estrans les plus développés.

\section{REMERCIEMENTS}

Nous exprimons nos vifs remerciements à Pierre Laplante, Denis Rouillard, Louis de Varennes, Richard Saint-Laurent, professeur et étudiants à l'université du Québec à Rimouski, ainsi qu'à Jean-Claude Dionne et Michel Allard, professeurs à l'université Laval (Québec), qui nous ont informé et accompagné sur le terrain, soit dans l'aire étudiée ici, soit dans la région de Québec pour y effectuer des comparaisons. Jean-Claude Dionne nous a en outre fourni d'indispensables et multiples données bibliographiques, parfois difficiles à trouver.

\section{REFERENCES}

BARTRUM, J.A. (1926): "Abnormal s shore platforms, J. Geol., vol. 34, p. $793-806$.

BIRD, J. B. (1964) : The Physiography of Arctic Canada, Baltimore, Johns Hopkins Press, $220 \mathrm{p}$.

BROCHU, M. (1961): Déplacement de blocs et d'autres sédiments par la glace sur les estrans du Saint-Laurent en amont de Québec, Ottawa, Min. Mines et Relevés tech., Etude géogr. $n^{\circ} 30,16 \mathrm{p}$.

CAILLEUX, A. et HAMELIN, L.-E. (1967) : Périglaciaire actuel sur le littoral du Bic (Bas-Saint-Laurent), Cah. Géogr. Qué., n 23, p. 361-378.

CHAMPAGNE, P. (1978) : Évolution littorale et dynamique glacielle à la pointe d'Argentenaye, tle d'Orléans, Québec, Univ. Laval, thèse M.A. (géographie), non publ., 133 p.
DALY, R. (1902): Geology of the northeast coast of Labrador, Bull. Harvard Comp. Zool., n' 38, p. 302-370.

DAWSON, A.G. (1980) : Shore erosion by frost: an example from the Scottish Lateglacial, in Studies in the Lateglacial of North-West Europe, J.S. HOUSE et al., édit., Oxford, Pergamon Press, p. 45-53.

DIONNE, J.-C. (1961): Quelques particularités de la morphologie littorale du Saint-Laurent, Rev. can. Geogr., vol. 15, nos $1-4$, p. $19-27$.

(1962): Note sur les blocs d'estran du littoral sud du Saint-Laurent, Can. Geogr., vol. 6, n² 2, p. 69-77.

- (1963a): Les types de cotes de la rive sud de l'estuaire du Saint-Laurent, Norois, $n^{\circ} 38$, p. 151-160.

(1963b) : Le probleme de la terrasse et de la falaise Mic Mac (cóte sud de l'estuaire maritime du Saint-Laurent), Rev. can. Géogr., vol. 17, $n^{\text {os }}$ 1-2, p. 9-25.

(1967) : Formes de corrosion littorale, côte sud du SaintLaurent, Cah. Géogr. Qué., nं 23, p. 379-395.

(1968): Morphologie et sedimentologie glacielles, cote sud du Saint-Laurent, Zeit. Geomorph., Sp. Bd. n 7, p. 5684.

(1969) : Erosion glacielle, estuaire du Saint-Laurent, Rev. Géogr. Montr., vol. 23, p. 5-20.

- (1970): Aspects morpho-sédimentologiques du glaciel, en particulier des côtes du Saint-Laurent, Univ. Paris (Sorbonne), thèse non publ., 2 vol., 412 p., et Québec, Env. Canada, Centre Rech. for. Laurentides, Rapp. Int. Q-F-X-9, $324 \mathrm{p}$.

(1972a): Caractéristiques des schorres des région froides en particulier dans l'estuaire du Saint-Laurent, Zeit. Geomorph., Suppl. Bd. 13, p. 131-162.

(1972b) : Les plates-formes rocheuses littorales de l'estuaire du Saint-Laurent, Ann. ACFAS, vol. 39, p. 76 et texte manuscrit, $9 \mathrm{p}$.

(1972c): Caractéristiques des blocs erratiques des rives de l'estuaire du Saint-Laurent, Rev. Géogr. Montr., vol. 26 , p. $125-152$.

(1973) : La notion de pied de glace (icefoot), en particulier dans l'estuaire du Saint-Laurent, Cah. Géogr. Qué. $n^{\circ} 41$, p. 221-250.

(1974) : Bibliographie annotée sur les aspects géologiques du glaciel, Québec, Env. Canada, CRFL, Rapp. Infor. LAU-X-9, $122 \mathrm{p}$.

(1977): La mer de Goldthwait au Québec, Geogr. phys. Quat., vol. 31, p. 61-80.

- (1979) : Les blocs d'estran a Saint-Fabien-sur-Mer, estuaire maritime du Saint-Laurent, Québec, Maritime Sed., vol. $15, n^{\circ} 1$, p. 5-13.

EDWARDS, A.B. (1951): Wave action in shore platforms erosion, Geol. Mag., vol. 88, p. 41-49.

GOLDTHWAIT, J.W. (1911): The twenty-foot terrace and seacliff of the Lower St. Lawrence, Am. J. Sci., vol. 32, p. 291317.

GUILCHER, A. (1952): Formes de décomposition chimique dans la zone des embruns et des marées sur les côtes bri- 
tanniques et bretonnes, 50 Anniv. Lab. Géogr. Univ. Rennes, Vol. Jubil., p. 167-181.

(1954): Morphologie littorale et sous-marine, Paris, P.U.F., 216 p.

(1980): Observations géomorphologiques sur des littoraux subarctiques de la pointe nord de I'ile Sakhaline (Extrème-Orient soviétique), Rev. Géomorph. dyn., vol. 29, p. 101-115.

HAMELIN, L.E. (1960): Classification générale des glaces flottantes. Naturaliste Can., vol. 87, n¹0, p. 209-227.

HILLS, E.S. (1949) : Shore platforms, Geol. Mag., vol. 86, p. 137. 152.

JOHNSON, D.W. (1925): The New England-Acadian shoreline, New York, Wiley, 608 p.

LAJOIE, J., HEROUX, Y. et MATHEY, B. (1974): The Precambrian shield and the Lower Paleozoic shelf: the unstable provenance of the Lower Paleozoic sandstones and conglome. rates of the Appalachians between Beaumont and Bic, Quebec, Can. J. Earth Sci., vol. 11, p. 951-963.

LAURIOL, B. et GRAY, J.T. (1980): Processes responsible for the concentration of boulders in the intertidal zone in Leaf Basin, Ungava, in The coastline of Canada, McCANN, S. B., édit., Geol. Surv. Can., Pap 80-10, p. 281-292.

LAUTRIDOU, J.-P. et COUTARD, J.-P. (1971): Recherches de gélifraction expérimentale du Centre de Géomorphologie. Schistes, grès et roches métamorphiques de BasseNormandie. Conclusions générales, Centre de Géomorph. du CNRS, Caen, $n^{\circ} 10,84$ p.

LOKEN, O. (1962): The Late-Glacial and Post-Glacial emergence and the deglaciation of northernmost Labrador, Geogr. Bull., $n^{\circ} 17$, p. 23-56.
LYELL, C. (1854): Principles of Geology, 1re ed., New York, Appleton, 834 p.; trad. fr. de la dern. ed., 1873, 2 vol., 921 et $847 p$.

POOLE, W.H., SANFORD, B.V., WILLIAMS, H. et KELLEY, D.G. (1970): Geology of southeastern Canada, in DOUGLAS, R.J.W., édit., Geology and economic minerals of Canada, p. 229-304.

ROSEN, P.S. (1979) : Boulder barricades in Central Labrador, J. Sed. Petrol, vol. 49, p. 1113-1124.

SLAUCITAJS, L. (1929): Spaltenbildung in der Eisdecke und Eisschiebungen an der Küste des Rigaschen Meerbusens im Winter 1928-29, Ann. d. Hydr. und Marit. Meteorol., vol. 57. p. $411-414$.

TANNER, V. (1939) : Om de blockrika strandgōrdlana vid subarktiska oceankustar, forekomstătt og upkomst, Terra, vol. 51 p. 157-165.

TRENHAILE, A.S. (1978) : The shore platforms of Gaspe, Québec. Ann. Amer. Geogr., vol, 68, p. 95-114.

TRENHAILE, A.S. et RUDAKAS, P.A. (1981): Freeze-thaw and shore platform development in Gaspé, Québec, Geogr. phys. Quat., vol. 35, nos 2-3, p. 171-181.

VILLENEUVE, G.O. (1967): Sommaire climatique du Quebec, Québec, Min. Rich. nat., Serv. Météo., Rapp. M-24, $168 \mathrm{p}$.

CARTES: Canada, Québec, 1/50 000, feuilles $22 \mathrm{C} / 7$ (Rimouski, $22 \mathrm{C} / 8$ (Sainte-Blandine), $22 \mathrm{C} / 9$ (Mont-Joli). Canada, Québec, $1 / 250000$, feuille 22 C (Rimouski).

PHOTOGRAPHIES AERIENNES: Ministère des Terres et Forêts, Service de la photogrammétrie et de la cartographie, photocartothèque provinciale, Québec, échelle: 1/15840, Q $63317-45$ à $72 ; 94$ à $98: Q 63316-210$ à 237. 